Cultures \& Conflits

28 | hiver 1997

Interpréter l'Europe

\title{
Une grammaire européenne de l'expérimentation sociale
}

Pierre Muller et Sophie Rouault

\section{(2) OpenEdition \\ 1 Journals}

Édition électronique

URL : http://journals.openedition.org/conflits/381

DOI : 10.4000/conflits.381

ISSN : 1777-5345

Éditeur :

CCLS - Centre d'études sur les conflits lilberté et sécurité, L'Harmattan

Édition imprimée

Date de publication : 15 janvier 1997

ISSN : 1157-996X

Référence électronique

Pierre Muller et Sophie Rouault, "Une grammaire européenne de l'expérimentation sociale », Cultures \& Conflits [En ligne], 28 | hiver 1997, mis en ligne le 16 mars 2006, consulté le 30 mars 2021. URL:

http://journals.openedition.org/conflits/381 ; DOI : https://doi.org/10.4000/conflits.381

Ce document a été généré automatiquement le 30 mars 2021.

Creative Commons License 


\title{
Une grammaire européenne de l'expérimentation sociale
}

\author{
Pierre Muller et Sophie Rouault
}

Pierre MULLER, Sophie ROUAULT ${ }^{1}$

2 Depuis quelques années, les études européennes semblent entamer un retour aux problématiques classiques de la science politique, recourant notamment à la méthode comparative, même si cette dernière est en partie inadéquate puisque s'attachant à la stateness d'un objet politique non étatique (au sens de l'Etat-Nation) produisant pourtant des politiques publiques légitimes. Cette approche ${ }^{2}$ permet notamment d'envisager l'intégration communautaire comme un exercice de créativité institutionnelle de longue haleine. Elle permet aussi de mettre davantage l'accent sur les interactions entre les divers niveaux de gouvernement (communautaire, nationaux, régionaux, locaux) à l'occasion de la formulation et de la mise en œuvre des politiques communautaires. Apparentées à un système de " gouvernance multi-niveaux "3, ne correspondant pas seulement aux mécanismes classiques de la régulation étatique ou hiérarchique mais aussi à des formes de coordination plus sociétales, plus informelles, les politiques de l'Union sont alors envisagées comme des actions collectives négociées et coordonnées, créant à leur tour des formes de légitimation nouvelles, ne correspondant plus nécessairement à celles attachées aux théories classiques de la représentation. Ce contexte polycentrique met également en évidence le fait que la définition des politiques publiques fait aujourd'hui l'objet d'une concurrence entre une multiplicité d'autorités publiques (ou para-publiques) légitimes - l'une d'entre elles étant la Commission européenne. S'intéresser à la mise en œuvre de ces politiques communautaires revient donc à cerner au plus près ces interactions entre niveaux de gouvernement mais aussi entre Etat et société, lorsqu'il s'agit de donner réalité aux décisions prises, où se produisent nombre d'interprétations, d'appropriations de la lettre de la décision pour adapter celles-ci à des réalités sociales, politiques et institutionnelles nationales, régionales et locales. La mise en œuvre d'une politique peut en effet s'apparenter analytiquement à un processus de médiation ${ }^{4}$ marqué par de multiples formes d'apprentissage collectif. Dans la mesure où cette médiation 
correspond à la fois à un processus de (re)construction du sens de l'action par les acteurs impliqués et à un processus de réorganisation des relations entre ceux-ci ; elle implique des apprentissages d'ordre cognitif et organisationnel, générant des changements dans les modes d'action publique mais aussi dans la répartition des rôles et des pouvoirs entre acteurs, dans leur interdépendance et leurs modes de légitimation. On se propose de développer ces hypothèses à partir de l'étude de la mise en œuvre en France de programmes communautaires d'insertion et de formation professionnelles. En raison des contraintes qu'ils imposent aux administrations et aux organismes de formation professionnelle impliqués dans leur mise en œuvre, ces programmes expérimentaux constituent pour l'observateur un révélateur du renouveau de l'action publique dans un contexte polycentrique. Ces politiques seront envisagées comme un exercice de créativité institutionnelle, mettant en interaction de multiples niveaux de gouvernement (au sens anglo-saxon du terme), mais aussi des acteurs administratifs et sociétaux dans un jeu complexe de gouvernance multiniveaux. C'est la complexité même de ce contexte d'action collective négociée, d'interaction entre organisations, entre systèmes d'action qui incite à s'attacher à la dimension relationnelle et dynamique des institutions, mais aussi au rôle des idées ${ }^{5}$ et des images permettant de donner de l'intelligibilité à un contexte de gouvernance polycentrique complexe. Le programme d'initiative communautaire Emploi On s'attachera ici à la mise en œuvre du programme d'initiative communautaire Emploi6 (ou PIC Emploi) issu, comme les autres programmes de ce type ${ }^{6}$, de la réforme des fonds structurels européens de 1988 qui avait permis à la Commission européenne de lancer un certain nombre de programmes expérimentaux (représentant environ 10\% des fonds). Financé exclusivement par le fonds social européen (FSE), Emploi est piloté par le DGV (" Emploi, relations industrielles et affaires sociales "), direction générale imposante et très segmentée ${ }^{7}$. Ces initiatives communautaires s'inscrivent dans la montée en puissance du pouvoir d'initiative de la Commission dans le cadre les fonds structurels $^{8}$ : la réforme qu'elle a initiée en 1988 s'apparente en effet à l'élaboration d'une nouvelle grammaire du développement régional, comprenant un certain nombre de principes obligatoires pour l'élaboration et la mise en œuvre de fonds venant en appui aux politiques nationales (" cadres communautaires d'appui ") et soutenant des programmes expérimentaux plus limités. Parmi ces principes se trouvent les obligations de concentration géographique des fonds (système de zonage par objectif), de programmation concertée et de co-financement (par les divers niveaux de " gouvernement ", incluant les collectivités locales, et visant la complémentarité des actions), du partenariat entre administrations (communautaire et nationales), l'obligation d'évaluation continue des actions, et enfin la reconnaissance d'un droit à la Commission de lancer ses propres actions innovantes - un droit qu'elle a de fait déjà exercé. La Commission présente alors ces initiatives comme un laboratoire de l'innovation sociale, destiné à pallier l'incapacité des Etats membres à se donner cette marge d'action face au poids des politiques établies. La Commission veut s'affirmer comme un recours face à l'inertie institutionnelle étatique, mais aussi plus largement et selon une rhétorique ouvertement politique visant à justifier son activisme dans le domaine des politiques sociales au sens large - offrir à la société civile une participation accrue à la définition de l'action publique, constituer un forum pour les débats publics, et enfin doter l'ensemble des acteurs impliqués d'une capacité d'anticipation ${ }^{9}$. Les programmes d'initiative communautaire voient ainsi la méthode des fonds structurels doublée d'un second registre, plus spécifique à leur dessein expérimental : les projets 
financés dans ce cadre le seront selon des axes prioritaires négociés entre la Commission et les gouvernements, sachant que ces projets devront être " innovants " et comporter des " partenariats transnationaux entre porteurs de projets ". Cette grammaire - entendue comme un ensemble de règles d'action publique à décliner selon des réalités nationales et locales plurielles - était d'autant plus contraignante qu'elle était au départ extrêmement floue hors de ces expressions clefs : le décideur ayant posé des objectifs et une méthodologie sans spécifier les moyens et les dispositifs institutionnels à emprunter pour les atteindre, les conditions de mise en œuvre nationale et locale étaient à inventer. Ces politiques s'apparentaient à un " contrat incomplet "10 d'expérimentation - relevant autant des politiques redistributives qu'incitatives - proposé aux Etats membres et aux acteurs du développement local et qui devait impliquer, pour être complété, des arrangements négociés et des réinterprétations, qui sont autant d'adaptations stratégiques et d'appropriations de la lettre de la décision initiale. Ce sont des interactions re-créatives que l'on se propose de retracer ici. On mettra d'abord l'accent sur les formes d'apprentissage organisationnel et opérationnel que recèle la mise en œuvre de ces politiques, puis sur les apprentissages cognitifs, sociaux et politiques qui dessinent des images renouvelées de l'action publique ainsi que des modes de reconnaissance et de légitimation des acteurs impliqués. L'expérimentation comme contrainte pour l'action: les apprentissages organisationnels dans la mise en œuvre de l'Initiative Emploi On s'attachera dans un premier temps à leur mise en œuvre nationale, puis à leur mise en œuvre régionale et locale, en tentant à chaque étape de s'attacher à la nature et aux implications du changement politique observé, sachant que la grammaire de l'expérimentation posée par la Commission représente un fort degré de contrainte : il s'agit ici de discerner les formes prises par l'adaptation d'un Etat Providence national à un contexte de gouvernance multi-niveaux, où il peut ne pas être l'auteur exclusif de son action sociale. Adopté à Bruxelles à la fin de l'année 1990, le programme d'initiative communautaire Emploi cherchait à promouvoir l'adaptation de certains groupes cibles aux nouvelles demandes du marché du travail (femmes, salariés en situation précaire, chômeurs de longue durée, jeunes), à travers le financement de projets locaux innovants (à l'échelle d'un territoire donné) et l'échange, par le biais de partenariats transnationaux, de savoir-faire entre les projets choisis dans les différents Etats membres. Cette règle du jeu et la méthodologie de l'action qui l'accompagnait (conformément aux principes de la réforme des fonds structurels de 1988) étant admises, les administrations nationales devaient mettre en place les structures administratives et les circuits financiers nécessaires à la mise en route de cette expérimentation. En France est ainsi créée, au sein du ministère des Affaires sociales, une " mission Fonds social européen " qui met en place, pour chaque programme, un comité de suivi, composé de fonctionnaires nationaux de différents ministères, des partenaires sociaux et d'un représentant de la Commission, se réunissant une à deux fois par an pour vérifier le respect des orientations négociées entre Paris et Bruxelles. Elle est aussi chargée d'apporter une assistance technique aux " porteurs de projets " afin de les aider dans le montage et l'évaluation du projet, tâche qu'elle délègue à une structure para-ministérielle. La mise en œuvre nationale des initiatives donne donc lieu à une adaptation institutionnelle à la marge du système administratif et de ses segmentations traditionnelles. Dans le cas du ministère des Affaires sociales, cela se traduit par la création d'une nouvelle segmentation (mission FSE) mais aussi par la délégation du suivi des dossiers à des organismes para-ministériels, effectuant ainsi 
une redistribution de l'expertise légitime. Tel est en effet l'élément le plus marquant de cette mise en œuvre : l'innovation et la flexibilité institutionnelles; nécessaires pour répondre aux exigences de l'expérimentation sociale proposée par la Commission, ont impliqué un mouvement d'adaptation par " expulsion ", l'inertie institutionnelle du ministère des Affaires sociales étant jugée d'avance trop grande par ses propres membres (et cette tradition de délégation existant déjà dans le domaine de la formation professionnelle). Le cas du ministère de l'Agriculture semble confirmer cette incapacité de l'administration française à s'adapter aux contraintes posées par la Commission : alors que, dans un premier temps, ce ministère a tenté de mettre en place des structures internes pour animer au niveau national ces programmes (dans ce cas il s'agissait du volet rural de l'initiative Euroform), il s'est trouvé lui aussi peu à peu obligé, face à la rigidité des structures financières ministérielles notamment, " d'expulser " à ses marges cette mission de médiation qu'il avait d'abord abritée, pour la reloger dans une association para-administrative en lui passant commande d'une mission d'animation à l'identique. La construction d'une expertise légitime par délégation La gestion quotidienne de l'initiative Emploi ${ }^{11}$ - de la constitution des dossiers à la pré-sélection jusqu'à l'évaluation des projets financés en passant par des publications - est donc confiée à une association loi 1901- qualifiée de " paraministérielle ": l'association Racine. Cette structure, qui ne bénéficie du statut d'association qu'à partir de 1992, avait été créée en 1988 pour gérer la montée en puissance des premiers programmes européens de formation professionnelle (Eurotecnet, Iris, Petra). Elle était composée de fonctionnaires en détachement, souvent de profil militant, et déjà familiers des problématiques européennes, qui voyaient dans celles-ci l'opportunité de privilégier des approches de la formation jugées en France marginales, atypiques, ou trop novatrices par rapport aux politiques pratiquées. Audelà de cette délégation, il s'avère que les véritables règles du jeu de l'expérimentation (par opposition aux grands principes d'action) furent élaborées par Racine, dans la mesure où les responsables de cette association ont été contraints, face à la lente montée des demandes de financement émanant du terrain, d'inventer en permanence des procédures pour compenser l'absence de directives européennes ou ministérielles précises. Se définissant par la gratuité des services techniques rendus aux candidats au financement, Racine est censée accomplir une mission de service public pour le compte du ministère des Affaires sociales. Pour autant, il est évident que cette création institutionnelle va se situer rapidement à la croisée de tous les circuits d'information et s'affirmer comme le lieu principal de transmission de l'information et surtout comme le centre de la production d'expertise légitime, le tout s'inscrivant dans un équilibre précaire combinant loyauté et autonomie à l'égard du ministère grâce à la maîtrise de la dimension supra-nationale de cette expérimentation sociale. L'intervention des régions et le choix de la déconcentration Les principes de co-financement et de partenariat institutionnel inscrits au contrat par la Commission européenne visait notamment une coordination plus grande entre échelons administratifs et niveaux d'autorités élues, autour des axes prioritaires choisis par l'Etat membre mais surtout autour de projets de développement local. Les régions et départements (Conseils Régionaux et Généraux) furent donc mis à contribution d'un point de vue financier, mais une consultation menée par la Commission, dans le cadre d'un Livre Vert sur les initiatives communautaires, pour asseoir la légitimité de ces expérimentations face au Conseil des Ministres révéla que cette contribution ne s'était accompagnée d'aucune information des collectivités territoriales, qui se trouvaient " sommées de participer à 
des programmes dont elles ignoraient la teneur " ${ }^{12}$. Cette adaptation de mauvaise volonté tient à ce que ces collectivités territoriales commençaient à peine à s'acclimater à la problématique générale des fonds structurels et envisageaient d'un mauvais œil les contraintes supplémentaires attachées à ces programmes expérimentaux au regard de la masse financière qu'ils représentaient. Dans un premier temps, la mise en œuvre locale de ces programmes ne permit donc pas qu'une régulation de nature étatique se mette en place à l'échelon local : celle-ci demeurait essentiellement centrale et déléguée, ce qui ne favorisa pas l'émergence d'images cohérentes et éclairantes de ces actions et généra une concurrence parmi les organismes postulant à la délégation. La mise en œuvre d'une seconde réforme des fonds structurels, initiée en 1993 par la Commission et prônant la régionalisation des transferts de fonds structurels européens, fut une tentative pour modifier cet état de fait. La France choisit pour sa part de déconcentrer et non de décentraliser ces fonds : ce choix marque la volonté du gouvernement de mener une offensive de " renationalisation " des fonds structurels, cadres communautaires d'appui et initiatives. Cette réorientation se traduisit par un renforcement du personnel de la mission FSE mais aussi de sa tutelle sur l'assistance technique Racine, qui obtint le monopole de l'assistance technique nationale aux projets et devint surtout assistance technique du ministère: cette reconfiguration des tutelles marque la volonté du ministère de redevenir le détenteur d'une expertise légitime, d'abord déléguée avec une bride tenue très lâche. Il semble que les services déconcentrés de l'Etat ne se sont pas saisis des PIC Emploi. A l'exception de quelques personnalités (dans les délégations régionales à la Formation professionnelle, les DRTEFP, et dans les services des Droits des femmes), désireuses d'élargir leur horizon professionnel ou soucieuses d'actualiser leurs compétences en prenant en charge des dossiers européens, les services semblent avoir mal accepté de s'adapter à la gestion des PIC, perçue par certains comme " une brimade ", du fait de la disproportion entre niveau de contrainte et volume financier. Ce qui a eu pour conséquence de couper encore plus l'administration par rapport au terrain, les services déconcentrés ne désirant généralement pas assurer le rôle de facilitateur et la remontée de l'information au niveau central. Ce choix de la déconcentration marqua aussi le statu quo quant au rôle assigné aux régions et aux départements, avec des tentatives pour simplement interdire, par voie de circulaire ministérielle, les relations directes entre les autorités infra-nationales et les services de la Commission et en ne " facilitant pas " les visites des membres de la Commission sur le terrain. Il s'avère néanmoins que les collectivités territoriales ont été mobilisées dans le cadre de ces expérimentations par le biais des porteurs de projets eux-mêmes, selon un schéma ascendant et non plus descendant qui correspondait d'ailleurs aux vues de la Commission européenne. Soucieux de pérenniser leurs activités et de s'assurer une reconnaissance politique sur leur territoire, les organismes de formation ont mené, surtout à partir de 1993, une campagne de lobbying et d'information pour recueillir les contreparties financières exigées par la Commission. Mais ils ont aussi exercé - pour les plus importants et les plus familiers des labyrinthes de l'action publique communautaire en tout cas - une forme de médiation entre les autorités présentes sur le territoire, qui étaient souvent en concurrence, pour instaurer entre elles un dialogue minimal sur le développement local et résoudre de manière plus informelle les conflits qui les opposaient autour de leur activité d'insertion et de formation professionnelle. Au-delà de leur adaptation organisationnelle au contexte européen, ces organismes ont donc pris part à l'adaptation des collectivités territoriales aux problématiques 
européennes. La fonction d'innovation des politiques européennes Cette prise d'initiative du " terrain " vis-à-vis des collectivités territoriales tient au fait que les politiques européennes de formation professionnelle et d'insertion sociale prennent souvent le relais des politiques nationales en ce qu'elles permettent de financer des aspects du parcours d'insertion-formation que ces dernières ne prennent pas en compte (diagnostic de territoire pour évaluer les emplois pour lesquels il existe une demande sociale, suivi personnalisé de projets personnels ou collectifs lors de leur montage...). Ce constat est fait par les responsables des ministères, qui reconnaissent leur incapacité à intégrer de telles innovations dans leur propres priorités. Ces politiques servent donc de " salle d'attente " à des actions publiques expérimentales en satisfaisant momentanément la demande des formateurs en matière de lutte contre l'exclusion. Cette difficulté à intégrer de nouvelles procédures dans les modes opératoires des administrations a eu plusieurs effets.

- Conséquences sur les procédures de mise en concurrence des organismes susceptibles de présenter des projets : les organismes de formation, établissements privés et publics ou associations, qui eurent en 1990 et en 1993 connaissance de ces programmes expérimentaux, furent informés pour la plupart par le biais de contacts informels au sein des ministères concernés. Face au manque de publicité ${ }^{13}$, les ministères se sont vus obligés de rechercher parmi leurs contacts de terrain les organismes susceptibles d'avoir le profil et les capacités organisationnelles pour monter un projet de cette ampleur. De ce fait, lors du premier appel d'offre de 1990, ils ont sélectionné une sorte d'élite constituée principalement d'acteurs associatifs, même si ce public s'est élargi, lors de la seconde vague des initiatives Emploi (1994-1999), à de nombreux organismes publics et mutualistes de formation ainsi qu'à des structures professionnelles (comme les chambres d'agriculture) souhaitant lancer de nouvelles formations; une substitution des financements communautaires aux financements nationaux dans le domaine de l'expérimentation semble s'effectuer dans le secteur de la formation professionnelle.

- De plus, cette incapacité ministérielle à intégrer ces expérimentations ${ }^{14}$ encourage chez les acteurs de terrain un recours direct auprès des services de la Commission européenne pour acquérir un surplus de légitimité (ainsi que de nouveaux financements) et pour, en retour, faire pression sur les autorités publiques françaises ayant compétence dans le domaine. Apparait ici nettement le fait que la définition des politiques sociales nationales est aujourd'hui l'objet d'une concurrence entre une multiplicité d'autorités publiques légitimes, la Commission européenne étant l'une de celles-ci. Les acteurs de terrain, conscients pour certains de la pluralité de ces sources de légitimation, se trouvent donc à leur tour engagés dans le jeu de la gouvernance multi-niveaux. La configuration des relations d'acteurs est donc en constante transformation et aucun acteur n'occupe de position dominante, malgré les tentatives maladroites du gouvernement français de canaliser les relations entre autorités infranationales et services de la Commission.

- Ce constat encourage également la Commission européenne à accroître le nombre de ces expérimentations et à les prolonger dans le temps au point de leur faire perdre leur nature proprement expérimentale, dans la mesure où des organismes voient se pérenniser les financements européens. Pour certains responsables européens, la Commission est acculée à un seuil d'inefficacité dans la mesure où, dans le but de satisfaire les demandes des acteurs du développement local, les initiatives sont devenues trop nombreuses pour préserver une gestion proche des projets locaux, les 
écrans séparant les niveaux de gouvernement s'opacifiant du fait de la multiplication des procédures. Les apprentissages organisationnels auxquels donnent lieu les initiatives Emploi font donc apparaître l'incapacité de l'administration française à compléter le contrat d'expérimentation sociale proposé par la Commission européenne en intégrant dans ses politiques les données issues de ces expérimentations. De ce fait, ils mettent à jour la nature concurrentielle de l'élaboration de nouvelles politiques sociales transversales - comme les politiques de lutte contre l'exclusion - au sens où celles-ci ne respectent pas la sectorisation et l'inertie institutionnelle de l'Etat Providence. L'Etat français ne joue pas véritablement le rôle de facilitateur dans l'élaboration des règles du jeu de l'expérimentation, déléguant ce rôle de droit et de fait. La reconfiguration des réseaux d'acteurs à l'œuvre dans ces politiques européennes lui échappe aussi pour partie. Se pose ici la question de la légitimation des acteurs publics dans un contexte de dispersion de l'autorité politique: si l'Etat se réserve ici le droit d'attribuer de la légitimité experte, de la représentativité à certains metteurs en œuvre accrédités, sans exercer de véritable autorité politique " hégémonique " sur l'action publique, ni se donner de rôle central, il n'est lui-même reconnu que comme partiellement légitime ${ }^{15}$. On peut donc se demander ce qu'il en est de sa capacité à élaborer des images de ces actions, porteuses de sens pour les acteurs de terrain, au-delà des normes (les principes de 1988, les axes d'action choisis) et des valeurs (la solidarité entre territoires) élaborées avec la Commission - c'est à dire à assumer le rôle de médiateur, à créer les conditions administratives et politiques de la définition d'un nouvel espace d'expression des intérêts sociaux. Apprentissages cognitifs et changements politiques dans la mise en œuvre des Initiatives Emploi Suivre la mise en œuvre nationale et locale des initiatives Emploi par les adaptations institutionnelles auxquelles elles donnent lieu nous a permis de voir quels groupes d'acteurs se sont principalement attachés à compléter le contrat d'expérimentation incomplet. On se demandera ici quels sont les acteurs capables, de par leur position dans ce contexte polycentrique d'action publique, d'élaborer des images porteuses de sens et dotées de force explicative. Gouvernance multi-niveaux et opacité A l'exception des circuits financiers (que l'on a peu adaptés aux contraintes de cette expérimentation, comme en témoignent les retards de paiement qui restent très importants), l'invention des règles quotidiennes de mise en œuvre des programmes revient à des organismes extérieurs au ministère, agissant sur délégation ou en soustraitance. Ces délégations ont eu pour conséquence évidente de diminuer encore la visibilité, pour les administrations nationales, de la mise en œuvre locale des projets financés. Cette observation est aussi valable, mais plus habituelle, pour les services de la Commission européenne, qui s'attachent essentiellement à la justification financière des expérimentations notamment par des contrôles sur place $(10 \%$ des projets furent contrôlés en France sur la période 1990-1994), leur justification politique s'effectuant par une délégation du suivi qualitatif des programmes à des consultants ou des universitaires chargés de faire " remonter " du terrain les informations utiles pour constituer un argumentaire pour la prise de décision en Conseil des ministres et au Parlement. Cette incapacité à intégrer les modes opératoires de la Commission se révèle pleinement dans les procédures d'évaluation des initiatives Emploi: alors que l'obligation d'évaluation (finale depuis 1990 et continue depuis 1993) du programme est inscrite dans le contrat négocié avec la Commission, aucune évaluation finale de la première vague de l'initiative Emploi (1990-1994) n'a été menée jusqu'à présent en France par les autorités nationales (même si le projet fut évoqué en 1994). Par ailleurs, 
et paradoxalement, sachant qu'une évaluation peut constituer une source d'autorité et de légitimation, la mission FSE ne reprend pas à son compte les nombreuses évaluations (quantitatives ou qualitatives) produites par la Commission, par Racine, ou même par la cellule d'animation du ministère de l'Agriculture, alors qu'elle tente de " renationaliser " la gestion des initiatives Emploi. En raison de cette opacité qui les éloigne du terrain, fonctionnaires français et responsables bruxellois doivent se contenter en pratique comme ils le reconnaissent d'ailleurs - d'élaborer des normes et des obligations pour les porteurs de projets, ce qui affaiblit fortement leur capacité à produire des images pouvant donner du sens à ces programmes et mettre en cohérence l'implication de différents niveaux de gouvernance en fixant la place des acteurs participant à la mise en œuvre. La mise en œuvre du PIC Emploi semble aussi faire l'objet d'une délégation de la mise en sens. " Mise en réseau " et production de sens Face aux délégations de mise en œuvre et de mise en sens effectuées par les administrations nationale et communautaire, semblent donc se structurer de nouvelles formes d'institutionnalisation des relations entre acteurs. A travers des relations plus informelles puisque traversant des institutions existantes, largement basées sur le volontariat et la réciprocité, les participants au programme tentent, par l'élaboration de circuits d'échange d'informations et d'expertise stabilisés, de diminuer l'opacité et la complexité de la gouvernance à plusieurs niveaux auxquels ils ont affaire tout en élaborant des images éclairantes des programmes dans lesquels ils sont impliqués : il s'agit pour eux de " se mettre en réseau "16. Cette expression recouvre une double réalité dans le cas des initiatives Emploi : elle désigne d'abord la relation qui peut exister entre porteurs de projets regroupés au niveau national ; elle désigne ensuite la tentative de stabiliser sur un territoire et autour d'un projet de développement local l'ensemble des acteurs, professionnels et politiques, devant permettre de retisser le lien local entre acteurs sociaux et politiques concernés. La mise en réseau nationale des porteurs de projets peut s'effectuer par deux biais: elle peut relever de l'initiative des porteurs de projets eux-mêmes, en étant portée par un organisme de formation à forte légitimité régionale et aux ressources importantes par exemple. C'est ainsi qu'en région PACA (Provence-Alpes-Côte d'Azur), sur l'initiative d'un organisme public de formation (un GRETA), s'est établi un réseau, piloté par le rectorat, destiné à rassembler l'ensemble des organismes de formation pratiquant l'échange européen et international de formateurs et de stagiaires; ces réseaux souvent destinés à " capter des fonds " par la construction de consortiums européens bénéficient d'une " mauvaise réputation car n'exerçant pas de mission de service public ". Cette mise en réseau peut aussi être l'œuvre des structures agissant par délégation des ministères: tentée par Racine dans le cadre de sa mission d'assistance technique aux porteurs de projets, mais abandonnée à la suite de la massification des projets, cette initiative est reprise par la cellule d'animation rurale du ministère de l'Agriculture (" Europea $\mathrm{FP}^{17}$ ") qui, après avoir fonctionné au sein de la Direction générale de l'enseignement et de la recherche, est désormais abritée au sein d'une association para-ministérielle et constituée par ses membres en réseau de porteurs de projets en milieu rural. Ce dispositif, créé pour assurer une véritable mission de service public, a pour objectif " d'établir des chaînes de compétences, des savoir-faire, des problèmes et des solutions ", à titre gratuit. Dans cet exemple, la confrontation des acteurs de terrain a lieu au cours de séminaires qui portent sur divers aspects de leurs projets (inscription dans un territoire, contenu pédagogique, échange transnational) et s'effectue à partir de grilles d'enquête préalablement communiquées par Europea FP à ces organismes. Autour de ces 
exercices, perçus d'abord comme une contrainte supplémentaire, semble se construire progressivement un espace de sens s'apparentant à une double cartographie du PIC Emploi : d'une part, celle rassemblant les divers territoires concernés par les projets collectifs représentés, d'autre part, celle rassemblant les niveaux de gouvernement impliqués, leurs services et leurs représentations sur les territoires. Les autoévaluations informelles des projets qui s'effectuent ainsi par prise de parole et confrontation font apparaître que chaque projet Emploi se construit souvent autour d'une médiation entre les acteurs du développement local présents sur un territoire, médiation le plus souvent sur l'initiative des organismes de formation eux-mêmes (cf. supra), plus rarement sur l'initiative d'un correspondant régional de l'Etat particulièrement implanté sur ce territoire. Il nous semble donc bien que, selon les conclusions d'Andy Smith ${ }^{18}$, " les fonds structurels renforcent la recomposition non pas d'un échelon territorial (en l'occurrence la région) mais des territoires ". L'action publique européenne a donc ici pour effet de remettre en question des pratiques et des représentations locales (souvent faites d'oppositions irréconciliables entre acteurs collectifs) pour les renouveler à la marge, par un mécanisme de comparaison notamment, dans le cadre d'échanges avec d'autres organismes de formation en Europe ou de comparaisons inter- ou même intra-régionales. De plus, ces séminaires ont parfois pour effet inattendu de recomposer autour du PIC Emploi une territorialité élargie, des projets proches géographiquement et s'ignorant jusque là, décidant de collaborer dans la complexité. La seconde cartographie qui s'élabore au cours de ces séminaires tient à l'échange d'informations entre des organismes inégalement informés sur les procédures à leur disposition pour mener à bien leur projet européen, ainsi qu'à la présence de fonctionnaires nationaux motivés et de chercheurs invités (désignés comme " personnes ressources "): la confrontation de ces diverses informations sur les procédures financières et administratives et les approches du développement local semblent permettre à la plupart des participants de composer une géographie mentale, institutionnelle, mais aussi symbolique et normative où leur projet trouve alors sa place dans le contexte d'une gouvernance complexe. La remise en cause partielle des représentations de l'Etat Providence Suivre la mise en œuvre de politiques communautaires par les apprentissages organisationnels et cognitifs qu'elles engendrent permet de visualiser la réorganisation des relations d'acteurs et les modifications des représentations sociales qui accompagnent celle-ci. Ainsi, dans le cas des initiatives Emploi, l'impossibilité pour les autorités nationales de garder en leur sein les services chargés de la mise en œuvre du contrat d'expérimentation semble avoir abouti à retirer à ces derniers la capacité à intégrer de nouvelles images du secteur des politiques sociales, liées à l'intégration communautaire, dans la tradition française de l'Etat Providence. Les images qui en découlent relèvent par conséquent plutôt d'une concurrence, mais aussi d'une complémentarité par défaut, entre les différentes autorités produisant les politiques d'insertion sociale et professionnelle. Ce travail de médiation et de cartographie cognitive déléguée permet aux porteurs de projets d'identifier les multiples niveaux de ressources financières et de légitimité à leur disposition, et les mécanismes de concurrence qui régissent leur interaction. Il devient alors évident, pour ces acteurs, que le système français d'Etat Providence n'est plus l'unique pourvoyeur de politiques sociales, au sens de pourvoyeur d'idées, d'abstractions (spécialité des euro-fonctionnaires) mais aussi de légitimité et de financements. Ces politiques sociales expérimentales permettent ainsi de souligner combien les politiques sociales sont, dans ce contexte de gouvernance sur plusieurs 
niveaux, l'objet d'une concurrence interinstitutionnelle et d'une pluralité d'expertises légitimes: c'est en ce sens que l'on peut appliquer ici la formule de Leibfried et Pierson $^{19}$, inspirée du fédéralisme, qui veut que l'intégration européenne peut être apparentée à un processus de " construction étatique compétitive " (competitive statebuilding) d'un type particulier. Les politiques sociales européennes participent donc à l'élaboration d'une économie mixte de la Providence, mêlant acteurs publics et parapublics à des organismes privés " agréés " dans leur mission de service public par une autorité qui n'est pas l'Etat national. De ce fait, la Commission européenne participe à la reconfiguration des Etats Providence européens, non seulement par la régulation administrative et juridictionnelle, mais aussi par des politiques incitatives, qui sont autant de laboratoires des nouvelles pratiques et politiques sociales. La gouvernance multi-niveaux comme laboratoire d'observation du changement Pour conclure, il faut évoquer la double exploration conceptuelle que peut permettre l'étude de la mise en œuvre des politiques européennes. Il nous semble que cette approche permet d'abord d'envisager le changement à partir de l'observation des interactions fortes entre systèmes d'action publique qu'impliquent les politiques européennes. Cette approche permet dans un second temps d'envisager le rôle des politiques européennes et des institutions communautaires dans les reconfigurations qui affectent aujourd'hui les espaces publics nationaux. Les politiques européennes comme expérimentation de la réforme des Etats-Providence? Il s'agit de cerner le changement à l'interaction entre institutions, c'est à dire à l'interaction entre des standards d'action légitimes, entre des ordres pluriels, régulant les relations Etat-société. Ces interactions peuvent ainsi donner lieu à des adaptations institutionnelles (ou des phénomènes de résistance au changement), mais aussi à l'émergence de nouvelles formes d'institutionnalisation des relations entre acteurs de la sphère publique. Il nous est ainsi apparu que les politiques sociales expérimentales proposées par la Commission européenne donnent lieu à deux phénomènes marquants : l'adaptation institutionnelle par expulsion et la constitution de réseaux d'acteurs de la mise en œuvre qui dessinent les contours d'une régulation infra-étatique, où aucun acteur dominant ne semble à même de proposer une image structurante de l'action publique. La faible adaptabilité d'une administration nationale trop sectorisée pour intégrer des politiques sociales trans-sectorielles, même expérimentales, implique des adaptations institutionnelles périphériques, avec la création de structures hybrides " para-ministérielles " souvent associatives et dotées d'une mission de service public, et la délégation de l'expertise légitime à ces structures externalisées. Les services de l'Etat, loin d'assumer les rôles de " courtier " et " d'éducateur "20, ne semblent plus pouvoir assurer les rôles d'informateur, de facilitateur ou de conciliateur, et encore moins celui de producteur d'images intelligibles pour l'action - sinon en se plaçant hors du strict cadre étatique à travers des relations de sous-traitance. Dès lors, l'administration n'assure plus qu'une mission de contrôle financier, au point qu'une initiative destinée à simplifier la gestion et l'évaluation des projets locaux par la distribution d'un logiciel commun est ressentie par les acteurs de terrain comme une simple volonté de reprise de contrôle, dans un domaine où l'Etat n'est plus perçu comme le lieu principal de légitimation de l'action. Un constat identique semble s'attacher aux services de la Commission : initiateurs de ce contrat incomplet d'expérimentation sociale, ils ne jouent plus qu'occasionnellement les courtiers, pour tenter d'esquisser un rééquilibrage dans l'intervention des autorités publiques, en privilégiant dans les partenariats institutionnels le rôle des régions par exemple. Ils apparaissent encore plus rarement comme des médiateurs dans la mesure 
où ils sont incapables - en raison de l'écran que constituent les administrations nationales - d'avoir eux-mêmes une vision d'ensemble des pratiques qu'ils initient. Dans une sorte de division forcée du travail intellectuel et politique, la Commission se voit cantonnée dans le rôle du fournisseur d'abstractions, d'idées combinant philosophie publique de la solidarité territoriale et guide programmatique et méthodologique du développement local, destinées à renouveler les pratiques sociales et l'action publique, c'est à dire à être traduites par les acteurs de sa mise en œuvre. La structuration de relations entre les acteurs de la mise en œuvre de ces politiques s'effectue alors à travers des " réseaux " multiples et interconnectés, traversant les institutions impliquées formellement dans ces politiques et rassemblant ponctuellement sur la base du volontariat des individus émanant de ces institutions. Il semble que le terme de réseau répond parfois, chez les acteurs impliqués, à cette attente de structuration d'un espace de sens autour de ces politiques perçues comme éminemment complexes et éclatées, et dont les institutions qui prétendent les coordonner (DGV, ministères...) offrent des images contradictoires et inconciliables. Ainsi, au-delà de structures perçues comme figées au sein desquelles on refuse de s'impliquer totalement, le réseau rassemble " les bonnes volontés " de provenance institutionnelle diverse qui, à tâtons, par la prise de parole et la confrontation des standards légitimes d'action que chacun représente, voient émerger des phénomènes de médiation collective, permettant d'élaborer une cartographie compréhensive d'une action publique multi-niveaux. C'est donc à un niveau infra-étatique que se produit une véritable médiation sociale et politique, que se dessinent des images raisonnablement cohérentes des politiques publiques mises en œuvre. Cet apprentissage collectif, cette éducation mutuelle, représentent une véritable médiation en ce qu'il offre du sens et organise les relations entre des acteurs participants qui étaient jusque là isolés et ne possédaient qu'une vision partielle de leur insertion dans la gouvernance européenne et de l'insertion de cette gouvernance dans leur territoire. Cette confrontation permet en outre de construire une identité de groupe, que l'on pourrait définir comme celle des " initiés " des programmes européens, de ceux qui maitrisent la complexité mais aussi savent s'adapter au changement inéluctable ou du moins acceptent d'interroger et de remettre en cause leur pratique. C'est dans le cadre de ces réseaux que semblent véritablement pouvoir être créées les conditions politiques de la définition d'un nouvel espace d'expression des intérêts sociaux. Le fait que ces réseaux envisagent parfois de se constituer en groupe de pression, pour donner légitimement de la voix dans l'élaboration et la réforme des politiques sociales semble confirmer cette hypothèse. $C^{\prime}$ 'est donc bien à ce niveau de gouvernance mixte (puisque comprenant acteurs publics, para-publics et privés) que peut s'observer le changement politique et de politique. Cette action collective, sous forme de réseau de formation de formateurs, a un impact sur les stratégies d'action choisies, parfois sur les choix stratégiques à effectuer, mais surtout sur les buts, les idées et images portées par les acteurs et les institutions impliquées. Plus largement, ces politiques expérimentales mettent en évidence la concurrence qui se joue entre niveaux de gouvernance pour la définition de nouvelles politiques sociales, ou du moins pour tenter, par l'expérimentation, de résoudre les nouveaux problèmes sociaux auxquels sont confrontés les Etats Providence: l'extension du chômage et de l'exclusion sociale. Ces politiques, qui doivent être saisies comme de véritables politiques sociales, puisque inscrites dans la régulation des externalités négatives de l'intégration communautaire par le grand marché, soulignent donc comment, à la marge des Etats Providence, se dessinent une " économie mixte de 
la Providence ", impliquant dans la définition et la mise en œuvre de l'action publique des autorités publiques concurrentes mais aussi des institutions privées agissant par délégation implicite ou explicite. Ces politiques européennes nous offrent finalement une image déconcertante du changement : c'est l'image d'une régulation infra-étatique de l'action sociale, où le changement est certes le produit de l'interaction entre institutions et entre une pluralité de standards d'action légitimes, mais surtout où la pression pour le changement de l'action publique, impulsé par la Commission européenne, produit des hybrides institutionnels interrogeant les recompositions à l'œuvre aujourd'hui entre les sphères de la société civile, de l'Etat et du marché. Vers la définition d'un espace public de proximité21 ? Le fait que la Commission européenne ait offert aux acteurs des politiques sociales nationales un contrat largement incomplet (celui de l'expérimentation du développement local par la réinsertion sociale et la formation professionnelle), a mis en évidence, à travers sa mise en œuvre, l'incapacité de ses services à élaborer un véritable espace de confrontation - ou du moins un espace qu'elle animerait - et par là-même de proposer un espace de visibilité et d'intelligibilité aux acteurs impliqués dans cette expérimentation (et encore moins aux citoyens dans leur ensemble). De même, l'incapacité des acteurs nationaux de la mise en œuvre (ministères) à s'adapter face aux contraintes posées par ce contrat, conduit à penser qu'aucun de ces deux niveaux de gouvernance ne semble à même d'initier un espace de libre expression et de confrontation, où ils pourraient faire œuvre de médiation, ni de construire une scène politique visible et intelligible par chacun, du moins sans en être presque irrémédiablement exclus de par les délégations multiples qu'ils effectuent observation de mauvaise augure s'il en est quant à l'émergence d'un espace public européen mais qui ne préjuge pas de l'émergence d'un espace européen d'action publique fragmenté22. Ce sont bien des " valeurs et des images par défaut "que propose dans ces politiques la Communauté européenne : l'espace de sens et de libre expression de ces politiques expérimentales est en effet construit à travers des réseaux largement locaux, ou du moins rassemblant des acteurs locaux et quelques fonctionnaires et élus " volontaires ", sur l'initiative de structures se situant à la marge de la régulation étatique, agissant dans le cadre d'une " délégation fonctionnelle du débat "23. Ces politiques expérimentales apparaissent comme un espace de conciliation des intérêts locaux et de recomposition du territoire de l'action, mais elles proposent aussi des alternatives à la régulation étatique du social, à travers l'intrusion de la gouvernance européenne dans les régulations sociales et la légitimation d'une économie mixte du Welfare. C'est à ce niveau local ou intermédiaire que se situent les instances médiatrices de ses politiques, et que se construit l'identité d'un groupe social probable, celui des initiés de la gouvernance complexe. C'est aussi à travers ces réseaux et par l'éclaircissement des difficultés et des échecs des metteurs en œuvre que s'élabore la cartographie et donc la visibilité de cette gouvernance multi-niveaux dans toute sa complexité. Observer l'intégration européenne par le biais des apprentissages organisationnels et cognitifs, par les processus de médiation qu'enclenche la mise en œuvre d'une politique par l'interaction entre plusieurs standards d'actions légitimes, nous permet de faire l'hypothèse de l'élaboration autour de ces politiques communautaires de développement local d'un " espace public de proximité ", entendu comme un espace d'expérimentation du " pouvoir agir de concert sur un territoire donné ${ }^{24}$, de réactivation en retour (et non descendante) de la régulation politique par une redistribution des expertises au sein de relais sociétaux. C'est bien par le développement local, par une sorte de détour par le territoire et la confrontation 
d'expertises plurielles que se comble partiellement le déficit démocratique dont souffre la Communauté européenne. On voit donc apparaître une alternative au piège de la décision jointe (ou joint decision-trap ${ }^{25}$ ) qui caractérise souvent les processus décisionnels communautaires et le système politique communautaire dans son ensemble. En effet, cette stratégie de l'expérimentation sociale met en évidence, à l'aide d'un prisme d'inspiration fédéraliste, une seconde option, qui serait celle d'actions multiples et indépendantes jouant comme autant de laboratoires locaux de la gouvernance sociale, situés à la marge d'Etats à forte inertie institutionnelle (en tout cas dans le cas français), permettant d'observer le changement politique autour de phénomènes d'adaptation créative. C'est bien cet espace d'action concurrente, ces processus de régulation étatique compétitive ${ }^{26}$ qu'il était important de mettre en lumière dans la mise en œuvre de ces politiques européennes.

\section{NOTES}

1. Chargés de recherche au CEVIPOF/CNRS.

2. A. Sbragia, Europolitics : Institutions and Policy-Making in the " New " European Community, Washington D.C., Brookings, 1992.

3. F.W. Scharpf, " Community and Autonomy: Multi-Level Policymaking in the European Union ", Journal of European Public Policy, nº 1, 1992, pp. 219-242 ; G. Marks et alii, Governance in the European Union, London, Sage, 1996.

4. P. Muller : " La mise en œuvre des politiques européennes " in F. d'Arcy, L. Rouban (dir.), De la Ve République à l'Europe, hommage à J. L. Quermonne, Paris, Presses de la FNSP, 1996.

5. Cf. M. Weir, "Ideas and the Politics of Bounded Innovation " in S. Steinmo, K. Thelen \& F. Longstreth (eds), Structuring Politics : Historical Institutionalism in Comparative Analysis, Cambridge University Press, 1992.

6. C'est le cas du progamme Leader destiné au développement rural. Cf. Andy Smith, L'Europe au miroir du local, les fonds structurels et les zones rurales en France, en Espagne et au Royaume-Uni, Paris, L'Harmattan, 1996.

7. D. Coombes, Politics and bureaucracy in the European Community : a portrait of the Commission, London, MacMillan, 1970 ; D. Collins, The operation of the European Social Fund, London \& Camberra, Croom Helm, 1983 ; L. Cram, " The European Commission as a Multi-Organisation : Social Policy and IT Policy in the EU ", Journal of European Public Policy, autonme 1994, pp. 195-217 ; L. Cram, " Calling the Tune without Paying the Piper? Social Policy Regulation : the Role of the Commission in EC Social Policy ", Policy and Politics, 21(2), 1993.

8. M. Keating \& B. Jones (eds), The European Union and the Regions, Oxford, Clarendon Press, 1995 ; L. Hooghe (ed.), Cohesion Policy and European Integration : Building MultiLevel Governance, Oxford University Press, 1996.

9. Cf. l'intervention d'Odile Quintin (membre de la DG V) au séminaire " Organisations internationales et régulations sociales " à l'I.E.P. de Paris, 23 et 24 Mai 1997 (actes à paraître). 
10. Expression empruntée à G. Majone, " Décisions publiques et délibération ", Revue Française de Science Politique, juillet-août 1994, pp. 579-598.

11. A l'exception du volet Now (femmes), que le service Droit des femmes du ministère des Affaires sociales décidera de gérer par lui-même, dans un certain isolement, jusqu'en 1995.

12. Phrase extraite d'un des courriers envoyés par des collectivités territoriales et des associations au ministère des Affaires sociales au moment de la consultation lancée en 1993 par la Commission avec son Livre Vert sur l'avenir des Initiatives Communautaires.

13. Au Portugal par exemple, l'appel à projet se fait par voie d'affichage public.

14. Intégration aux politiques nationales que la Commission européenne qualifie de "mainstreaming" et fixe comme objectif à ces politiques expérimentales.

15. P. Durand, J.C. Thoenig, " L'Etat et la gestion publique territoriale ", Revue Française de Science Politique, vol. 46, n 4, août 1996.

16. On ne s'attachera pas ici aux débats académiques sur les réseaux (policy networks), mais plutôt aux fonctions cognitives attribuées par leurs membres à cette forme choisie d'institutionnalisation de leurs relations : il s'agit de voir en quoi cette dénomination fait sens pour ces acteurs. Les expressions entre guillemets contenues dans les paragraphes qui suivent sont à attribuer à des porteurs de projets, rencontrés lors de séminaires organisés pour le ministère de l'Agriculture dans le cadre d'une observation participante.

17. F.P. pour formation professionnelle.

18. Op. cit., p. 155.

19. S. Leibfried, P. Pierson (eds), European Social Policy, Between Fragmentation and Integration, Washington D.C., Brookings, 1995.

20. J.G. March et J.P. Olsen, " The New Institutionalism : Organizational Factors in Political Life ", American Political Science Review, Sept. 1984, pp. 734-79.

21. Sylvie Biarez, " Repenser la sphère locale selon l'espace public ", Contribution au IVe Congrès de l'AFSP, Aix-en-Provence, 1996.

22. Pierre Muller, " Un espace européen des politiques publiques " in Y. Mény, P. Muller, J.-L. Quermonne (dir.), Politiques publiques en Europe, Paris, L'Harmattan, 1995.

23. Sylvie Biarez, op. cit.

24. Ibid.

25. Fritz W. Scharpf : " The joint decision trap ", Public administration, vol. 66, autumn 1988, pp. 239-278 : le processus décisionnel communautaire générerait systématiquement des décisions correspondant à un plus petit dénominateur commun, au détriment d'une européanisation plus poussée des politiques publiques et malgré la médiation de la Commission européenne.

26. D'après l'expression de " competive state building " de Leibfried et Pierson, op. cit. 
INDEX

Index géographique : Bruxelles, Europe, Union européenne

Mots-clés : politiques publiques, bureaucraties/ théorie des organisations, construction européenne 